\title{
Nondestructive Detection of Authenticity of Thai Jasmine Rice Using Multispectral Imaging
}

\author{
Wei Liu $\mathbb{D}^{1,2}$ Xue Xu ${ }^{1},^{3}$ Changhong Liu ${ }^{1}{ }^{1}$ and Lei Zheng $\mathbb{D}^{1}$ \\ ${ }^{1}$ School of Food and Biological Engineering, Hefei University of Technology, Hefei 230009, China \\ ${ }^{2}$ Intelligent Control and Compute Vision Lab, Hefei University, Hefei 230601, China \\ ${ }^{3}$ Rice Research Institute, Anhui Academy of Agricultural Sciences, Hefei 230031, China \\ Correspondence should be addressed to Changhong Liu; liuchanghong1982@163.com and Lei Zheng; zhengleihf@163.com
}

Received 7 October 2020; Revised 28 December 2020; Accepted 5 January 2021; Published 20 January 2021

Academic Editor: Alessandro Di Cerbo

Copyright (@ 2021 Wei Liu et al. This is an open access article distributed under the Creative Commons Attribution License, which permits unrestricted use, distribution, and reproduction in any medium, provided the original work is properly cited.

\begin{abstract}
The detection of authenticity is essential to the development and management of Thai jasmine rice industry. In this study, the multispectral imaging system $(405-970 \mathrm{~nm})$ was used for the detection of adulteration in Thai jasmine rice combined with chemometric methods including principal component analysis (PCA), partial least squares (PLS), least squares-support vector machines (LS-SVM), and backpropagation neural network (BPNN). Three varieties of rice that were similar to Thai jasmine rice in appearance were selected to perform the classification and quantitative prediction experiments by multispectral images. For the classification experiment, four varieties of rice samples could be easily classified with accuracy achieved to $92 \%$ by the BPNN model. For the quantitative prediction of adulteration proportion experiments, the results showed that, among the different chemometric methods, LS-SVM achieved the best prediction performance comparing the results of coefficient of determination, root-mean-square error (RMSEP), bias, and residual predictive deviation (RPD). It can be concluded that multispectral imaging technology with chemometric methods can be applied in the rapid and nondestructive detection of authenticity of Thai jasmine rice.
\end{abstract}

\section{Introduction}

Rice (Oryza sativa), as one of the most important food crops, supplies staple foods for more than half of the world's population [1-3]. In the world rice market, Thailand is the largest rice exporting country over the past decade according to USDA [4], and Thai jasmine rice is one of the most popular varieties that command a premium price due to its unique flavor and delicious taste [5]. However, in recent years, Thai jasmine rice is vulnerable adulterated by mislabeling or part adulterated with other varieties of rice for higher commercial profits. Thus, how to detect authenticity of Thai jasmine rice fast and accurately is becoming a key issue to be solved in the rice industry to protect the interests of quality conscious consumers, stakeholders, and importing countries [6].

Till now, many methods were employed to detect the authenticity of rice, such as DNA fingerprinting [7, 8], fluorescence-based inter-simple sequence repeat-polymerase chain reaction (ISSR-PCR) and simple sequence repeats (SSR) markers [9], fluorescent simple sequence length polymorphisms [10], denaturing high-performance liquid chromatography [11], and high-resolution inductively coupled plasma mass spectrometry [12]. However, these traditional analytical methods are generally time-consuming, laborious, sophisticating, destructive, or toxic. Thus, it is necessary to develop a rapid and nondestructive method to ensure the quality of Thai jasmine rice in market.

With the improvement of the optical and spectroscopic techniques, some new methods were developed for rapid determination of food quality such as X-ray fluorescence [13], Raman spectroscopy [14], near infrared (NIR) spectroscopy [15-17], hyperspectral imaging [18-20], and $\mathrm{THz}$ spectroscopy [21]. Multispectral imaging, with the spectra range from visible light to near infrared, is regarded suitable for the rice detection because it can acquire both spatial and 
spectral information by combining the benefits of traditional imaging and spectroscopic techniques. Moreover, in multispectral system, some typical spectral bands were selected to avoid mass spectral imaging data which was caused by the high resolution of spectroscopy in NIR spectral and hyperspectral methods. Recently, this technique was applied in many agriculture fields such as discrimination of transgenic rice seeds and soybean seeds [22, 23], identification of tomato varieties [24], differentiation of soybean and weed species [25], detection of minced beef adulteration [26, 27], and detection of dicyandiamide in infant formula powder [28]. Meanwhile, to analyze the spectral data, some chemometric methods have been developed such as partial least square (PLS), least square-support vector machine (LSSVM), and backpropagation neural network (BPNN), which are important for the application of optical and spectroscopic techniques in nondestructive detection [29, 30].

To study the authenticity of Thai jasmine rice, the classification and adulteration experiments were developed using multispectral imaging technique combined with chemometric methods. Three varieties of rice that were similar to Thai jasmine rice in appearance were selected to perform the classification and quantitative prediction experiments. Comparing the results from different chemometric methods, the best model for the detection of authenticity of Thai jasmine rice was obtained.

\section{Materials and Methods}

2.1. Samples Preparation. Four varieties of rice including Thai jasmine rice, jasmine sticky rice, Simiao rice, and Northeast Wuchang rice were purchased from Metro supermarket of Hefei City, China (Figure 1). These four varieties of rice were similar in shape and color with the naked eye and stored in sealed plastic bags at $4^{\circ} \mathrm{C}$ until use.

Firstly, in classification experiment, a total of 800 intact rice samples (200 samples in each variety) were collected. Among all samples, 600 rice samples (150 samples in each variety) were randomly allocated to the calibration set, and 200 rice samples (50 samples in each variety) were randomly allocated to the prediction set. Then in adulteration experiment, Thai jasmine rice was selected as unadulterated rice samples and parts of them were adulterated with different proportions of other three varieties of rice, respectively. Rice samples including eleven adulterated proportions ranging from $0 \%$ to $100 \%(\mathrm{w} / \mathrm{w})(0 \%, 10 \%, 20 \%$, $30 \%, 40 \%, 50 \%, 60 \%, 70 \%, 80 \%, 90 \%$, and $100 \%)$ were prepared and each adulterated sample totally contained about $2 \mathrm{~g}$ of rice. In each adulteration experiment, there were 20 samples for each adulterated proportion and all samples summed up to 220 in total $(15 \times 11$ samples in calibration set and $5 \times 11$ samples in prediction set, respectively).

2.2. Multispectral Imaging System and Image Acquisition. VideometerLab instrument (Videometer A/S, Hørsholm, Denmark) with 19 different wavelengths ranging from $405 \mathrm{~nm}$ to $970 \mathrm{~nm}$ was used to capture multispectral images of rice samples. The detailed wavelengths were 405, 435, 450,
$470,505,525,570,590,630,645,660,700,780,850,870,890$, 910,940 , and $970 \mathrm{~nm}$. The instrument provides a rapid and nondestructive assessment of uniform and nonhomogeneous samples without preparation. The multispectral imaging system integrates illumination, a monochrome gray scale CCD camera, and computer technology with advanced digital image analysis and statistics. Spatial resolution of the multispectral images was $2056 \times 2056$. The images of rice samples without the background can be used for the extraction of spectra data. Moreover, morphological features including area $\left(\mathrm{mm}^{2}\right)$, length/width, roundness, and Hunter $L^{*}, a^{*}$, and $b^{*}$ values of rice were all extracted from the image analysis and processing. Detail information of the setup can be found in previous report [31]. Figure 2 shows the procedures of the authenticity detection of Thai jasmine rice.

2.3. Chemometrics Methods. Principal component analysis (PCA) is a method known for reducing the dimensionality of the system which is widely used to deal with large datasets like spectral data. It is possible to demonstrate differences between the various objects with fewer variables. In this study, PCA was used to distinguish the Thai jasmine rice from the other varieties of rice. With the multispectral imaging data, PCA can provide the most important information about the potential capability of differentiating the samples.

PLS, as a linear regression method for multivariate calibration, is used to find the fundamental relations between the predictors $(X)$ and the responses $(Y)$. Thus, it can reduce the primordial predictors to a new variable set called latent variables (LVs), which have the best predictive ability. The number of LVs, as a critical parameter in the algorithm, was determined by minimizing the value of the prediction residual error sum of square (PRESS).

Support vector machine (SVM) is an important classification learning algorithm. To make SVM suitable for engineering applications, researchers developed LS-SVM by applying least squares errors as the training error function and made it powerful for solving problems in nonlinear classification, function estimation, and density estimation. This modification simplifies the training process of SVM and reduces computation time [32]. Radial basis function (RBF) was used as the kernel function of LSSVM. The algorithm comprises two main parameters: one is a regularization parameter that determines the trade-off between structural and empirical risk minimization and affects the generalization performance of the LS-SVM model, and the other represents the radial width of the kernel function. The details of LS-SVM algorithm could be found in the literature [33].

BPNN is also a common model utilized in agricultural products detection studies, which can solve complex problems more accurately than linear techniques. In this study, BPNN has a three-layer network consisting of an input layer, a hidden layer, and an output layer. Leave-oneout cross-validation procedure was used during the calibration step. Several network architectures were tested by varying the number of neurons in the hidden layer with 


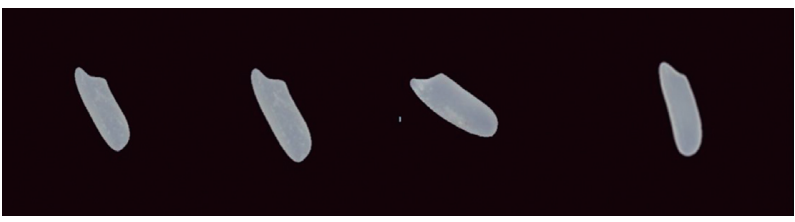

(a)

(b)

(c)

(d)

Figure 1: Images of the four varieties of rice. (a) Thai jasmine, (b) jasmine sticky, (c) Simiao, and (d) Northeast Wuchang.

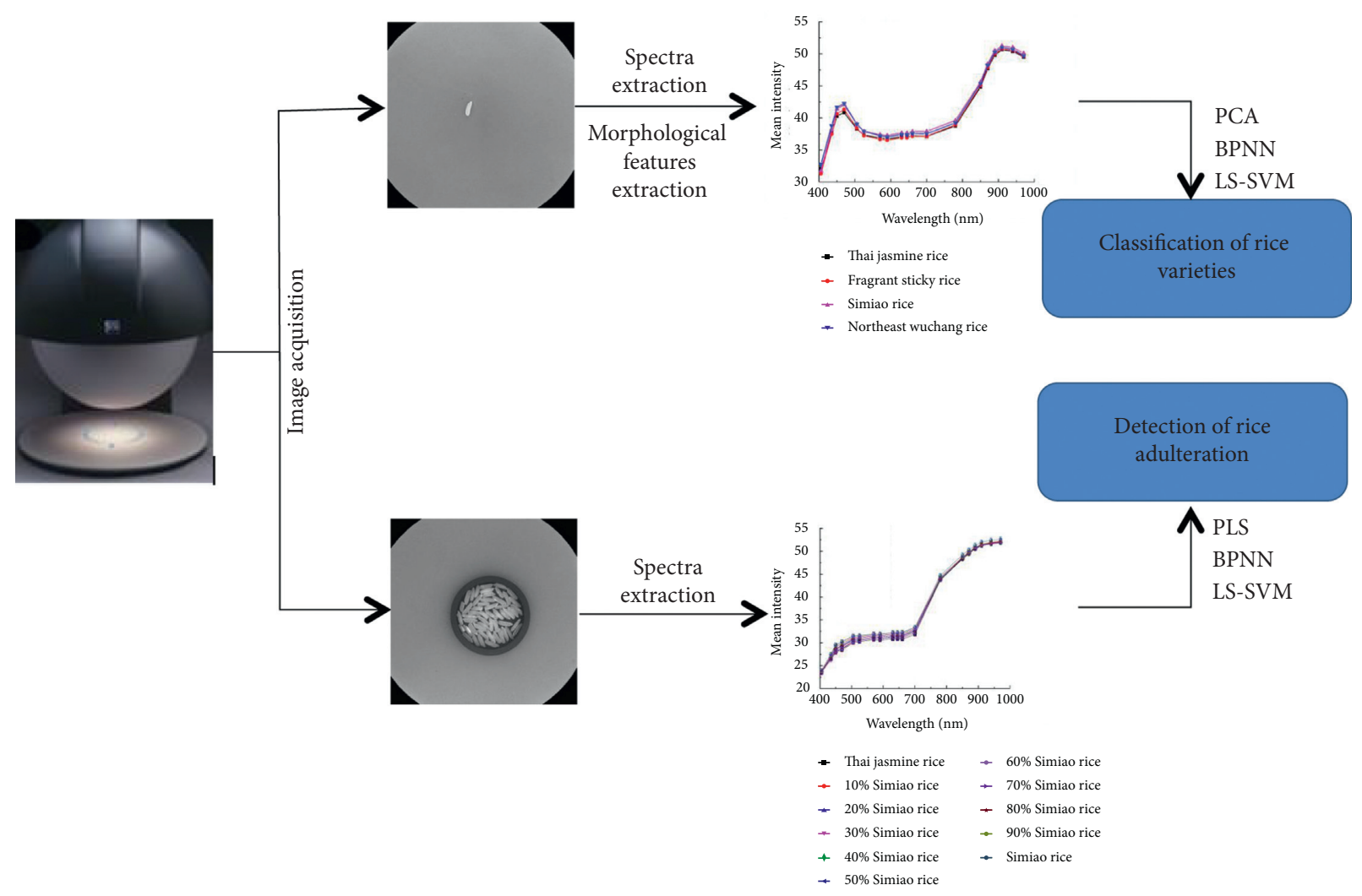

FIGURE 2: Flowchart of main steps for authenticity detection of Thai jasmine rice using multispectral imaging.

different initial weights. The optimal parameters such as hidden nodes, the goal error, and iteration times were determined by the least prediction error. The theory of BPNN has been described in detail in a previous publication [34].

2.4. Evaluation of Model Performance. For the analysis of spectral data in experiments, the multivariate data must be trained to build a calibration model and then evaluated for its performance with prediction set of samples. The analysis model was evaluated with the parameters of root mean square error of calibration (RMSEC), root mean square error of prediction (RMSEP), bias, and the coefficient of determination $\left(R^{2}\right)$ in calibration $\left(R_{\mathrm{C}}^{2}\right)$ and prediction $\left(R_{\mathrm{P}}^{2}\right)$. Moreover, the residual predictive deviation (RPD) was used to evaluate how well a calibration model can predict in new prediction samples. High RPD value indicates very good prediction in samples outside the calibration set. Generally, an optimum model should have high values of $R_{\mathrm{C}}^{2}, R_{\mathrm{P}}^{2}$ and RPD, and low values of RMSEC and RMSEP.
2.5. Statistical Analysis. All statistical analyses for multispectral imaging features data of the four different varieties of rice samples, including area $\left(\mathrm{mm}^{2}\right)$, length/width, roundness, and Hunter $L^{*}, a^{*}$, and $b^{*}$ were performed using SAS software. The data were expressed as mean \pm standard deviation and analyzed with one-way analysis of variance (ANOVA). Duncan's multiple range test was used for mean comparison.

\section{Results and Discussion}

3.1. Analysis of Multispectral Images. Table 1 shows the features of the four different varieties of rice samples, including area $\left(\mathrm{mm}^{2}\right)$, length/width, roundness, and Hunter $L^{*}, a^{*}$, and $b^{*}$. In these parameters, the morphology of the rice could be seen from area, roundness, and length/width values and the color of the rice could be seen from the Hunter $L^{*}, a^{*}$, and $b^{*}$ values. From the statistical analysis in Table 1, it can be seen that the Northeast Wuchang rice was the most different variety among the four varieties of rice 
TABle 1: Analysis of imaging features for four different varieties of rice.

\begin{tabular}{lcccccc}
\hline Variety & Area $/ \mathrm{mm}^{2}$ & Length/width & Roundness $/ \mathrm{mm}$ & $L^{*}$ & $a^{*}$ \\
\hline Thai jasmine rice & $3.07 \pm 0.34 \mathrm{~b}$ & $12.78 \pm 1.10 \mathrm{a}$ & $2.57 \pm 0.27 \mathrm{~b}$ & $67.18 \pm 1.50 \mathrm{~b}$ & $-0.25 \pm 0.14 \mathrm{~b}$ & $-4.55 \pm 1.13 \mathrm{a}$ \\
Jasmine sticky rice & $3.48 \pm 0.27 \mathrm{a}$ & $12.34 \pm 0.83 \mathrm{~b}$ & $2.76 \pm 0.25 \mathrm{a}$ & $67.19 \pm 0.98 \mathrm{~b}$ & $-0.27 \pm 0.12 \mathrm{~b}$ & $-4.57 \pm 1.09 \mathrm{a}$ \\
Simiao rice & $3.06 \pm 0.24 \mathrm{~b}$ & $12.54 \pm 1.21 \mathrm{~b}$ & $2.54 \pm 0.22 \mathrm{~b}$ & $67.61 \pm 1.90 \mathrm{a}$ & $-0.14 \pm 0.13 \mathrm{a}$ & $-4.60 \pm 1.05 \mathrm{~b}$ \\
Northeast Wuchang rice & $2.80 \pm 0.14 \mathrm{c}$ & $12.20 \pm 0.95 \mathrm{c}$ & $2.29 \pm 0.19 \mathrm{c}$ & $67.61 \pm 2.03 \mathrm{a}$ & $-0.26 \pm 0.15 \mathrm{~b}$ & $-5.33 \pm 1.13 \mathrm{c}$ \\
\hline
\end{tabular}

Data correspond to the means \pm standard error of two hundred rice samples. Different small letters in the same column indicate significant difference among different varieties of rice samples $(P<0.05)$.

samples in appearance. The Thai jasmine rice and Simiao rice were similar in length/width values, while there was no significant difference between Thai jasmine rice and jasmine sticky rice in CIE $L^{*}, a^{*}$, and $b^{*}$.

Average reflection spectra of rice samples in each variety are shown in Figure 3. It could be seen that the general trend of spectra of the four varieties of rice samples was very similar. Although the shape and color were similar among the four varieties of rice with the naked eyes, apparent difference could be observed around $450 \mathrm{~nm}$ that was related to blue-green color. In Table $1, \mathrm{CIE} L^{*}, a^{*}$, and $b^{*}$ values of different varieties of rice were given. $L^{*}$ is used to describe the lightness, $a^{*}$ presents the redness or greenness, and $b^{*}$ designates the blueness. It could be seen that there was significant difference in $b^{*}$ values among the four varieties of rice, which was consistent with the result in reflection spectra in Figure 3. Meanwhile, there was no obvious difference in reflection intensity of NIR region especially between 940 and $970 \mathrm{~nm}$ that was high related to the water content of the detection object [35]. Comparing the results of $L^{*}, a^{*}$, and $b^{*}$ in Table 1 and the reflection spectra values in Figure 3, it could be seen that the difference in color was more apparent than in water content among the four varieties of rice. This may be due to the similar water content in all varieties of rice.

3.2. Analysis of PCA. PCA was performed initially to examine the qualitative difference of Thai jasmine rice and other three varieties of rice in principal component (PC) space. Figure 4 shows the three-dimensional principal component score plot using the first three score vectors, and PC1, PC2, and PC3 were derived from spectral features, morphological features, and combination of spectral and morphological features of the rice samples, respectively. The first three factors accounted for all spectral data information of $99.18 \%, 99.63 \%$, and $99.59 \%$ for three different features combination, which showed that PC1, PC2, and PC3 could present almost all the information of the spectral data.

The results in Figures 4(a) and 4(b) suggested that it could not separate the four varieties of rice clearly only with spectral or morphological features, while in Figure 4(c), the discrimination between Thai jasmine rice and other varieties of rice was more apparent than in Figures 4(a) and 4(b). The results suggested that both spectral features and morphological features were key factors for the discrimination of Thai jasmine rice and other varieties of rice. However, it could not be used to classify the four varieties of samples

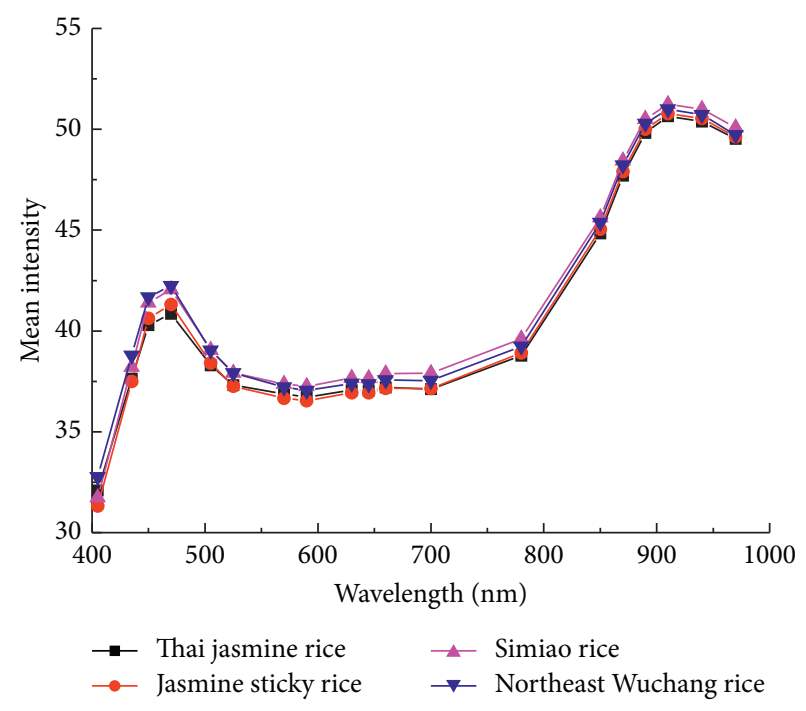

FIgURE 3: Average reflection spectra from the multispectral images of Thai jasmine rice and three other varieties of rice.

directly due to the overlaps among four varieties of rice. Thus, the advanced intelligent chemometric methods were used to obtain the discrimination model with both the spectral and morphological features in this study.

\subsection{Classification of Different Varieties of Rice.} Establishment of an accurate and reliable predictive model for determining rice varieties was necessary to discriminate the Thai jasmine rice. The LS-SVM and BPNN prediction models were built by using the spectral and morphological features data. Table 2 shows the discrimination results of four varieties of rice in the calibration set and prediction set. In the prediction set, the classification accuracies of LS-SVM and BPNN were $82 \%$ and $89 \%$ with the spectral data, respectively. Meanwhile, the classification accuracies of LSSVM and BPNN were achieved in $84 \%$ and $92 \%$ with the combined spectral and morphological features, respectively. These results proved that the discrimination performance could be improved by using the combination of spectral and morphological features. Meanwhile, compared with LSSVM, BPNN was a better chemometric method for discriminating Thai jasmine rice from other three varieties of rice with the accuracy being 100\% using the combined spectral and morphological features in the calibration set, while in the prediction set, the discrimination performance was $92 \%$ with the combined spectral and morphological features data. 


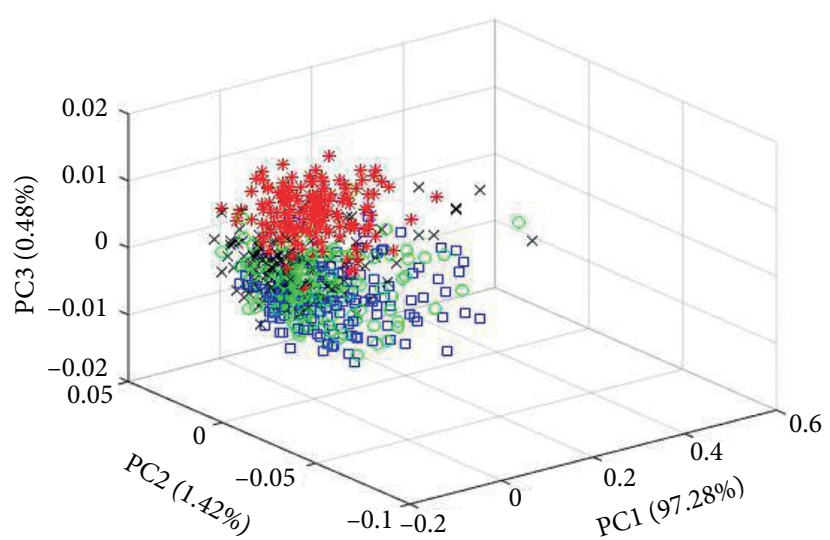

(a)

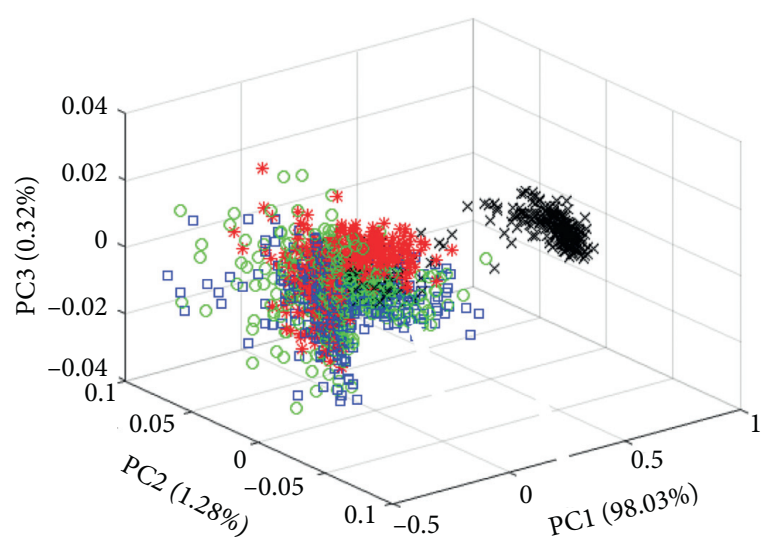

(b)

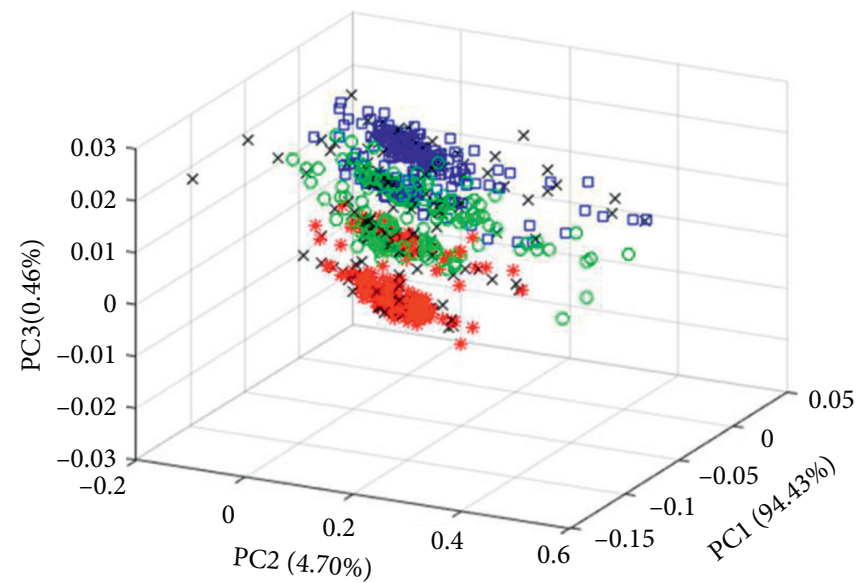

(c)

Figure 4: Three-dimensional score plots of the first three principal components derived from spectral features (a), morphological features (b), and combination of spectral and morphological features (c), respectively. The dots in plots represented Thai jasmine rice $\left({ }^{*}\right)$, jasmine sticky rice $(\mathrm{O})$, Simiao rice $(\square)$, and Northeast Wuchang rice $(\times)$.

3.4. Quantitative Detection of Adulteration. Quantitative detection models were obtained using PLS, BPNN, and LS-SVM methods. The performances of these three models for predicting Thai jasmine rice proportions are shown in Table 3 . The excellent prediction results for the three different varieties of rice were achieved in adulteration experiments. Most of the coefficients of determination of the results for each method were beyond 0.90. In general, the results of the Thai jasmine rice adulterated with Northeast Wuchang rice was the best in both determination and root mean square error, which showed that it was the most different variety from Thai jasmine rice, while the lowest values of $R_{\mathrm{C}}^{2}$ and $R_{\mathrm{p}}^{2}$ were found in the results of adulteration with the jasmine sticky rice which showed it may be the most similar variety to Thai jasmine rice. For the variety of Northeast Wuchang rice, the best detection model was obtained using LS-SVM with $R_{\mathrm{C}}^{2}$ of $0.995, R_{\mathrm{P}}^{2}$ of 0.988 , RMSEC of $1.398 \%$, bias of $2.10 \%$, RMSEP of $1.603 \%$, and the RPD of 8.482 . For the most similar variety of jasmine sticky rice, the best detection model was obtained using LS-SVM with $R_{\mathrm{C}}^{2}$ of $0.990, R_{\mathrm{P}}^{2}$ of 0.935 , RMSEC of $2.164 \%$, bias of $0.382 \%$, RMSEP of $2.582 \%$, and the RPD of 9.674 .
Furthermore, the RPD value showed that the LS-SVM model was adequate for predicting the adulteration proportion of Thai jasmine rice.

For the chemometrics methods, there were apparently different effects for each method to Thai jasmine rice detection. Compared with BPNN and PLS models, the LSSVM model had the best predictive results with all $R_{\mathrm{C}}^{2}$ and $R_{\mathrm{P}}^{2}$ being over 0.93 in three different adulterated varieties of rice. Although some values of $R_{\mathrm{C}}^{2}$ and $R_{\mathrm{P}}^{2}$ in PLS and BPNN models were similar with LS-SVM model, the RMSEP was higher than those in LS-SVM model. The results indicated that the LS-SVM model was more suitable for adulteration proportion prediction of Thai jasmine rice compared to PLS and BPNN.

Different varieties of rice may have different physical and chemical properties, which can be used to achieve the variety classification through chemometric methods and spectral imaging [36]. Thus, although different varieties of rice samples were similar in appearance to the human eye, there were some differences in the special spectral band which can be related in visible and NIR spectral reflectance [3]. Chemometric methods can highlight the differences among different varieties of samples and reduce 
TABLE 2: Comparison of discrimination performance obtained with LS-SVM and BPNN methods with the spectral data and the combined spectral and morphological features data.

\begin{tabular}{|c|c|c|c|c|}
\hline & \multicolumn{2}{|c|}{ LS-SVM } & \multicolumn{2}{|c|}{ BPNN } \\
\hline & MS & Accuracy (\%) & MS & Accuracy (\%) \\
\hline \multicolumn{5}{|l|}{ Calibration set } \\
\hline \multicolumn{5}{|l|}{ Thai jasmine rice $(n=150)$} \\
\hline Spectral & 4 & 97.3 & 0 & 100 \\
\hline Spectral + morphology & 4 & 97.3 & 0 & 100 \\
\hline \multicolumn{5}{|l|}{ Jasmine sticky rice $(n=150)$} \\
\hline Spectral & 33 & 78 & 1 & 99.3 \\
\hline Spectral + morphology & 34 & 77.3 & 0 & 100 \\
\hline \multicolumn{5}{|l|}{ Simiao rice $(n=150)$} \\
\hline Spectral & 14 & 90.6 & 0 & 100 \\
\hline Spectral + morphology & 14 & 90.6 & 0 & 100 \\
\hline \multicolumn{5}{|c|}{ Northeast Wuchang rice $(n=150)$} \\
\hline Spectral & 4 & 97.3 & 0 & 100 \\
\hline Spectral + morphology & 4 & 97.3 & 0 & 100 \\
\hline \multicolumn{5}{|l|}{ Total } \\
\hline Spectral & 55 & 90.8 & 1 & 99.8 \\
\hline Spectral + morphology & 56 & 90.6 & 0 & 100 \\
\hline \multicolumn{5}{|l|}{ Prediction set } \\
\hline \multicolumn{5}{|l|}{ Thai jasmine rice $(n=50)$} \\
\hline Spectral & 2 & 96 & 2 & 96 \\
\hline Spectral + morphology & 1 & 98 & 2 & 96 \\
\hline \multicolumn{5}{|l|}{ Jasmine sticky rice $(n=50)$} \\
\hline Spectral & 18 & 64 & 6 & 88 \\
\hline Spectral + morphology & 10 & 80 & 3 & 94 \\
\hline \multicolumn{5}{|l|}{ Simiao rice $(n=50)$} \\
\hline Spectral & 11 & 78 & 4 & 92 \\
\hline Spectral + morphology & 12 & 76 & 7 & 86 \\
\hline \multicolumn{5}{|c|}{ Northeast Wuchang rice $(n=50)$} \\
\hline Spectral & 5 & 90 & 10 & 80 \\
\hline Spectral + morphology & 9 & 82 & 4 & 92 \\
\hline \multicolumn{5}{|l|}{ Total } \\
\hline Spectral & 36 & 82 & 22 & 89 \\
\hline Spectral + morphology & 32 & 84 & 16 & 92 \\
\hline
\end{tabular}

MS: misclassified samples.

TABLE 3: Performance of PLS, LS-SVM, and BPNN models for predicting adulteration proportion in Thai jasmine rice (\%).

\begin{tabular}{lccccccc}
\hline Variety & Chemometrics & $R_{\mathrm{C}}^{2}$ & RMSEC (\%) & $R_{\mathrm{P}}^{2}$ & Bias & RMSEP (\%) & RPD \\
\hline \multirow{3}{*}{ Jasmine sticky rice } & PLS & 0.946 & 3.741 & 0.873 & 0.792 & 4.679 & 4.753 \\
& BPNN & 0.982 & 2.893 & 0.923 & 0.681 & 3.827 & 6.826 \\
& LS-SVM & 0.990 & 2.164 & 0.935 & 0.382 & 2.582 \\
Simiao rice & PLS & 0.983 & 6.259 & 0.984 & 0.730 & 5.780 & 5.567 \\
& BPNN & 0.988 & 4.731 & 0.984 & 0.971 & 5.842 & 5.477 \\
\hline \multirow{3}{*}{ Northeast Wuchang rice } & LS-SVM & 0.991 & 3.961 & 0.983 & -0.465 & 3.569 & 8.791 \\
& PLS & 0.981 & 2.924 & 0.933 & 2.04 & 1.10 & 9.033 \\
& BPNN & 0.981 & 1.906 & 0.980 & 3.61 & 1.813 & 6.528 \\
& LS-SVM & 0.995 & 1.398 & 0.988 & 2.10 & 1.603 \\
\hline
\end{tabular}

$R_{\mathrm{C}}^{2}$, coefficient of determination in calibration; $R_{\mathrm{P}}^{2}$, coefficient of determination in prediction; RMSEC, root mean square error of calibration; RMSEP, root mean square error of prediction; RPD, residual predictive deviation.

the variation. In this study, the result was similar to a previous study [23] in that the spectral data and the morphological features data were both key factors for the rapid discrimination of different varieties of rice seeds.
The prediction results confirmed the suitability of multispectral imaging for determining adulteration proportion in Thai jasmine rice in a rapid and nondestructive manner. 


\section{Conclusion}

The detection of authenticity of Thai jasmine rice using multispectral imaging combined with chemometrics methods was investigated through the classification and adulteration experiments. The results showed that both spectral data and morphological data were key factors for the rapid screening of Thai jasmine rice. Thai jasmine rice could be discriminated from other varieties of rice even if they were similar to the human eyes. Furthermore, quantitative prediction of adulterated rice proportion in Thai jasmine rice could be achieved. The best results were obtained by LSSVM combined with spectral and morphological features. In conclusion, the present study has shown that the use of multispectral imaging combined with chemometric methods for detection of authenticity of Thai jasmine rice was a very attractive platform and had the potential to be widely used in rapid and on-site screening because it is nondestructive, simple, and without pretreatments.

\section{Data Availability}

Data are available upon request to the corresponding author.

\section{Conflicts of Interest}

The authors have no conflicts of interest.

\section{Authors' Contributions}

Wei Liu contributed to the study design, collected the data, interpreted the results, and reviewed the manuscript. Lei Zheng designed the study, interpreted the results, and drafted the manuscript. Changhong Liu analyzed the multispectral imaging data and reviewed the manuscript. Xue $\mathrm{Xu}$ collected rice samples and multispectral imaging data.

\section{Acknowledgments}

This work was supported by the National Key R\&D Program of China (2020YFC1606802), the Key Science \& Technology Specific Projects of Anhui Province (202003a06020022 and 18030701200), the Natural Science Foundation of Anhui Province of China (2008085MC96 and 1908085QF270), the Fundamental Research Funds for the Central Universities (JZ2019YYPY0027), and the Talent Research Foundation of Hefei University (18-19RC43).

\section{References}

[1] X. Feng, Q. Zhang, P. Cong, and Z. Zhu, "Preliminary study on classification of rice and detection of paraffin in the adulterated samples by Raman spectroscopy combined with multivariate analysis," Talanta, vol. 115, pp. 548-555, 2013.

[2] X. Hu, W. Lang, W. Liu, X. Xu, J. Yang, and L. Zheng, "A nondestructive terahertz spectroscopy-based method for transgenic rice seed discrimination via sparse representation," Journal of Infrared, Millimeter, and Terahertz Waves, vol. 38, no. 8, pp. 980-991, 2017.

[3] W. Liu, C. Liu, F. Ma, X. Lu, J. Yang, and L. Zheng, "Online variety discrimination of rice seeds using multispectral imaging and chemometric methods," Journal of Applied Spectroscopy, vol. 82, no. 6, pp. 993-999, 2016.

[4] E. Robinson, USDA Estimating Record Global Rice and Soybean crops, Delta Farm Press Exclusive Insight, New York, NY, USA, 2013.

[5] S. Wongpornchai, K. Dumri, S. Jongkaewwattana, and B. Siri, "Effects of drying methods and storage time on the aroma and milling quality of rice (Oryza sativa L.) cv. Khao Dawk Mali 105," Food Chemistry, vol. 87, no. 3, pp. 407-414, 2004.

[6] A. Gonzalvez, S. Armenta, and M. De La Guardia, "Traceelement composition and stable-isotope ratio for discrimination of foods with protected designation of origin," $\operatorname{Tr} A C$ Trends in Analytical Chemistry, vol. 28, no. 11, pp. 1295-1311, 2009.

[7] P. R. Choudhury, S. Kohli, K. Srinivasan, T. Mohapatra, and R. P. Sharma, "Identification and classification of aromatic rices based on DNA fingerprinting," Euphytica, vol. 118, no. 3, pp. 243-251, 2001.

[8] X. Ren, X. Zhu, M. Warndorff, P. Bucheli, and Q. Shu, "DNA extraction and fingerprinting of commercial rice cereal products," Food Research International, vol. 39, no. 4, pp. 433-439, 2006.

[9] J. Nagaraju, M. Kathirvel, R. R. Kumar, E. A. Siddiq, and S. E. Hasnain, "Genetic analysis of traditional and evolved Basmati and non-Basmati rice varieties by using fluorescencebased ISSR-PCR and SSR markers," Proceedings of the National Academy of Sciences USA, vol. 99, no. 9, pp. 5836-5841, 2002.

[10] H. F. J. Bligh, "Detection of adulteration of Basmati rice with non-premium long-grain rice," International Journal of Food Science and Technology, vol. 35, no. 3, pp. 257-265, 2000.

[11] Z. Peng, X. Yuan, Y. Huang et al., "Application of denaturing high-performance liquid chromatography for rice variety identification and seed purity assessment," Molecular Breeding, vol. 36, no. 19, pp. 1-14, 2016.

[12] P. Cheajesadagul, C. Arnaudguilhem, J. Shiowatana, A. Siripinyanond, and J. Szpunar, "Discrimination of geographical origin of rice based on multi-element fingerprinting by high resolution inductively coupled plasma mass spectrometry," Food Chemistry, vol. 141, no. 4, pp. 3504-3509, 2013.

[13] T. Noda, S. Tsuda, M. Mori et al., "Determination of the phosphorus content in potato starch using an energy-dispersive X-ray fluorescence method," Food Chemistry, vol. 95, no. 4, pp. 632-637, 2006.

[14] M.-L. Xu, Y. Gao, X. X. Han, and B. Zhao, "Detection of pesticide residues in food using surface-enhanced Raman spectroscopy: a review," Journal of Agricultural and Food Chemistry, vol. 65, no. 32, pp. 6719-6726, 2017.

[15] M. M. Oliveira, J. P. Cruz-Tirado, J. V. Roque, R. F. Teófilo, and D. F. Barbin, "Portable near-infrared spectroscopy for rapid authentication of adulterated paprika powder," Journal of Food Composition and Analysis, vol. 87, Article ID 103403, 2020.

[16] W. G. C. Bandara, G. W. K. Prabhath, D. W. S. C. B. Dissanayake, V. R. Herath, G. M. R. I. Godaliyadda, M. P. B. Ekanayake et al., "Validation of multispectral imaging for the detection of selected adulterants in turmeric samples," Journal of Food Engineering, vol. 266, Article ID 109700, 2020.

[17] J. Y. Barnaby, T. D. Huggins, H. Lee, A. M. McClung, S. R. M. Pinson, M Oh et al., "Vis/NIR hyperspectral imaging distinguishes sub-population, production environment, and physicochemical grain properties in rice," Scientific Reports, vol. 10, p. 9284, 2020. 
[18] Z. Gao, Y. Shao, G. Xuan, Y. Wang, Y. Liu, and X. Han, "Realtime hyperspectral imaging for the in-field estimation of strawberry ripeness with deep learning," Artificial Intelligence in Agriculture, vol. 4, pp. 31-38, 2020.

[19] Z. Gao, Y. Zhao, L. R. Khot, G. A. Hoheisel, and Q. Zhang, "Optical sensing for early spring freeze related blueberry bud damage detection: hyperspectral imaging for salient spectral wavelengths identification," Computers and Electronics in Agriculture, vol. 167, Article ID 105025, 2019.

[20] D. Liu, Y. Wu, Z. Gao, and Y.-H. Yun, "Comparative nondestructive classification of partial waxy wheats using nearinfrared and Raman spectroscopy," Crop and Pasture Science, vol. 70, no. 5, pp. 437-441, 2019.

[21] K. Wang, D.-W. Sun, and H. Pu, "Emerging non-destructive terahertz spectroscopic imaging technique: principle and applications in the agri-food industry," Trends in Food Science \& Technology, vol. 67, pp. 93-105, 2017.

[22] C. Liu, W. Liu, X. Lu, W. Chen, J. Yang, and L. Zheng, "Nondestructive determination of transgenic Bacillus thuringiensis rice seeds (Oryza sativa L.) using multispectral imaging and chemometric methods," Food Chemistry, vol. 153, pp. 87-93, 2014.

[23] C. Liu, W. Liu, X. Lu, W. Chen, F. Chen, J. Yang et al., "Nondestructive discrimination of conventional and glyphosateresistant soybean seeds and their hybrid descendants using multispectral imaging and chemometric methods," The Journal of Agricultural Science, vol. 154, no. 1, pp. 1-12, 2016.

[24] S. Shrestha, L. Deleuran, M. Olesen, and R. Gislum, "Use of multispectral imaging in varietal identification of tomato," Sensors, vol. 15, no. 2, pp. 4496-4512, 2015.

[25] C. J. Gray, D. R. Shaw, P. D. Gerard, and L. M. Bruce, "Utility of multispectral imagery for soybean and weed species differentiation," Weed Technology, vol. 22, no. 4, pp. 713-718, 2008.

[26] A. I. Ropodi, D. E. Pavlidis, F. Mohareb, E. Z. Panagou, and G.-J. E. Nychas, "Multispectral image analysis approach to detect adulteration of beef and pork in raw meats," Food Research International, vol. 67, pp. 12-18, 2015.

[27] A. I. Ropodi, E. Z. Panagou, and G.-J. E. Nychas, "Multispectral imaging (MSI): a promising method for the detection of minced beef adulteration with horsemeat," Food Control, vol. 73, pp. 57-63, 2017.

[28] C. Liu, W. Liu, J. Yang, Y. Chen, and L. Zheng, "Non-destructive detection of dicyandiamide in infant formula powder using multi-spectral imaging coupled with chemometrics," Journal of the Science of Food and Agriculture, vol. 97, no. 7, pp. 2094-2099, 2017.

[29] Y.-Z. Feng and D.-W. Sun, "Application of hyperspectral imaging in food safety inspection and control: a review," Critical Reviews in Food Science and Nutrition, vol. 52, no. 11, pp. 1039-1058, 2012.

[30] H. Huang, L. Liu, and M. Ngadi, "Recent developments in hyperspectral imaging for assessment of food quality and safety," Sensors, vol. 14, no. 4, pp. 7248-7276, 2014.

[31] M. S. Andresen, B. S. Dissing, and H. Løje, "Quality assessment of butter cookies applying multispectral imaging," Food Science \& Nutrition, vol. 1, no. 4, pp. 315-323, 2013.

[32] Z. Su, B. Tang, Z. Liu, and Y. Qin, "Multi-fault diagnosis for rotating machinery based on orthogonal supervised linear local tangent space alignment and least square support vector machine," Neurocomputing, vol. 157, pp. 208-222, 2015.

[33] A. Borin, M. F. Ferrão, C. Mello, D. A. Maretto, and R. J. Poppi, "Least-squares support vector machines and near infrared spectroscopy for quantification of common adulterants in powdered milk," Analytica Chimica Acta, vol. 579, no. 1, pp. 25-32, 2006.

[34] H. Dai and C. Macbeth, "Effects of learning parameters on learning procedure and performance of a BPNN," Neural Networks, vol. 10, no. 8, pp. 1505-1521, 1997.

[35] D. Wu, Y. He, and S. Feng, "Short-wave near-infrared spectroscopy analysis of major compounds in milk powder and wavelength assignment," Analytica Chimica Acta, vol. 610, no. 2, pp. 232-242, 2008.

[36] X. Zhang, F. Liu, Y. He, and X. Li, “Application of hyperspectral Imaging and chemometric calibrations for variety discrimination of maize seeds," Sensors, vol. 12, no. 12, pp. 17234-17246, 2016. 